\title{
Detection of electron and hole traps in CdZnTe radiation detectors by thermoelectric emission spectroscopy and thermally stimulated conductivity
}

\author{
E. Y. Lee ${ }^{a}$, B. A. Brunett ${ }^{b}$, R. W. Olsen ${ }^{a}$, J. M. Van Scyoc III', H. Hermon ${ }^{a}$, R. B. James ${ }^{a}$ \\ aSandia National Laboratories, Advanced Materials Research Dept., Livermore, CA 94551 \\ ${ }^{b}$ Carnegie Mellon University, Dept. of Electrical and Computer Engineering, Pittsburgh, PA 15213 \\ 'University of California, Dept. of Materials Science and Engineering, Los Angeles, CA 90095
}

\begin{abstract}
The electrical properties of CdZnTe radiation detectors are largely determined by the electron and hole traps in this material. The traps, in addition to degrading the detector performance, can function as dopants and determine the resistivity of the material. Thermoelectric emission spectroscopy and thermally stimulated conductivity are used to detect these traps in a commercially available spectrometer-grade $\mathrm{CdZnTe}$ detector, and the electrical resistivity is measured as a function of temperature. A deep electron trap having an energy of $695 \mathrm{meV}$ and cross section of $8 \times 10^{-16} \mathrm{~cm}^{2}$ is detected and three hole traps having energies of $70 \pm 20 \mathrm{meV}, 105 \pm 30 \mathrm{meV}$ and $694 \pm 162 \mathrm{meV}$ are detected. A simple model based on these traps explains quantitatively all the data, including the electrical properties at room temperature and also their temperature dependence.
\end{abstract}

Keywords: CdZnTe, radiation detector, $\mathrm{x}$-ray detector, $\gamma$-ray detector, trap, compensation, TSC, TEES, current-voltage, semiconductor radiation detector

\section{INTRODUCTION}

Recently, CdZnTe x-ray and $\gamma$-ray detectors have received much attention due to their potential applications, including identification of nuclear materials and imaging for medical and security purposes'. However, the materials issues are still poorly understood. For example, although it is commonly observed that these detectors show effects of trapping, degrading the energy resolution of $x$-ray and $\gamma$-ray spectrometers, it is not known what impurities or defects cause the trapping. Also, it is observed that the resistivity of these materials can approach that for an intrinsic material, but, despite these impurities or defects, the compensation mechanism that must be responsible for this behavior is not known.

Thermoelectric emission spectroscopy ${ }^{2 \cdot 6}$ and thermally stimulated conductivity ${ }^{7}$ are two related methods to detect traps and to infer their energies, cross sections, and concentrations. We use these methods to characterize the traps in commericially available $\mathrm{CdZnTe}$ detectors. Based on the results, we construct models consisting of the observed trap levels in the material, and use the models to predict the electrical behaviors of the detectors. We also perform variable temperature current-voltage measurements and compare the behavior predicted by the models to the actual electrical behavior observed. This way, the validity of the model is checked.

In this paper, we discuss the results from a sample obtained from a commercial supplier of spectrometer-grade $\mathrm{CdZnTe}$ detectors. The sample was grown by the high-pressure Bridgeman method and its electrical properties are representative of some of the samples we have studied. A good agreement between all the experimental data and the model is found, with only the trap concentrations as variable parameters.

\section{EXPERIMENTAL SETUP AND PROCEDURE}

The electrical measurements are performed inside a closed-cycle He cryostat by Janis Research Company, Inc., in which the sample temperature can vary between $14 \mathrm{~K}$ and $475 \mathrm{~K}$. The cryostat is continuously pumped by a custom ordered turbopump-mechanical pump system made by Varian Vacuum Products, to maintain a pressure in the order of

whisubutibod mannowipt hes been anfhored by 8 contreator of the Unitod States Govemament ander controct. Acoordingly the Unitod States Gov" crmont rotains a non-exclustiv, zoyalty-freo license to publish or ro. produce the published form of this cortuibrtion, or allow others to do so, 


\section{DISCLAIMER}

This report was prepared as an account of work sponsored by an agency of the United States Government. Neither the United States Government nor any agency thereof, nor any of their employees, make any warranty, express or implied, or assumes any legal liability or responsibility for the accuracy, completeness, or usefuiness of any information, apparatus, product, or process disclosed, or represents that its use would not infringe privately owned rights. Reference herein to any specific commercial product, process, or service by trade name, trademark, manufacturer, or otherwise does not necessarily constitute or imply its endorsement, recommendation, or favoring by the United States Government or any agency thereof. The views and opinions of authors expressed herein do not necessarily state or reflect those of the United States Government or any agency thereof. 


\section{DISCLAIMER}

Portions of this document may be illegible in electronic image products. Images are produced from the best available original document. 
$10^{-7}$ Torr, and the cryostat is fixed to an optical table. A metallic box is placed over the cryostat during measurements, to eliminate stray light and to shield electrical noise.

The sample stage in the cryostat is home-made, and it consists of a spring loaded sample holder and mirrors to pipe the light from the cryostat viewport down to the sample. The spring loading is necessary to keep the sample stationary and also to provide good thermal and electrical contacts to the sample through out the temperature range from $14 \mathrm{~K}$ to $475 \mathrm{~K}$. The sample is typically a parallelepiped piece of CdZnTe with Au electrodes on the top and the bottom surfaces, and the sample is placed flat on the sample holder. A spring loaded Teflon piece holds the sample down. For photoexcitation at low temperature, the sample is illuminated from the side. External to the cryostat, a $250 \mathrm{~W}$ tungstenhalogen lamp is used as a light source. The light is first passed through two lenses to collect and focus the light, then it enters a McPherson monochromator that ranges from 200 to $1000 \mathrm{~nm}$. The output of the monochromator is collected by a fiber optic bundle that pipes the light into the view port of the cryostat. The two planar mirrors on the sample holder guides the light to the sample. After passing through the monochromator, the light has a bandwidth of $\sim 5 \mathrm{~nm}$ FWHM, as measured independently using a second spectrometer.

Two silicon diode sensors are placed at the bottom and the top of the sample to measure the temperatures at the two electrodes on the sample. An electrical wire is connected each of the electrodes, to enable biasing of the sample and to measure current. These wires go out of the cryostat via a feedthrough and they are connect by a triaxial cable to an electrometer, Keithley 6517A, to measure currents between $\sim 1 \mathrm{~mA}$ and $\sim 1 \mathrm{fA}$. The temperature is measured by a Lakeshore temperature controller, DRC-93CA, with precision of $\sim 0.1 \mathrm{~K}$, and it also controls the cryostat temperature. Both the Keithley and the Lakeshore apparatuses are interfaced by GPIB to a PC compatible computer, and a home-made LabVIEW software is used for automated control and measurement. The setup for our experiment is shown below in Fig. 1.

\section{variable temperature cryostat ( $14 \mathrm{~K}$ to $475 \mathrm{~K}$ )}

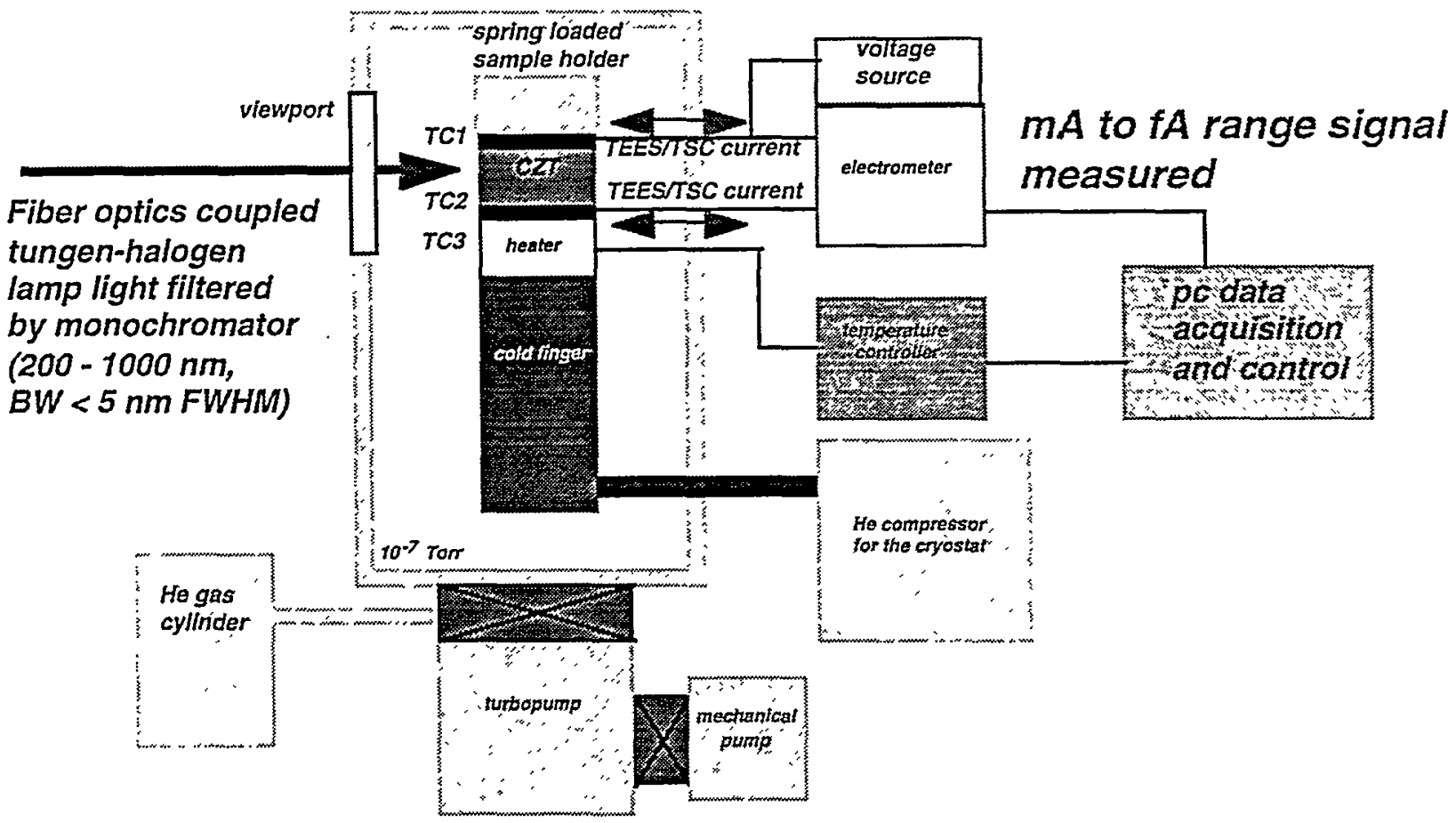

Fig. 1. The setup for TEES, TSC, and current-voltage measurements.

For the current-voltage measurement, the following is the experimental procedure. First, to load a new sample, the metallic box over the cryostat is removed, the vacuum pumps are shut down, cryostat is vented to air, and it is 
opened. The sample is inserted into the spring loaded sample holder, and the cryostat is closed and the vacuum pumps are started. The metallic box is placed over the cryostat and sufficient time is allowed for the pressure inside the cryostat to reach high vacuum. Then the temperature of the sample is recorded, a voltage bias is applied, and the current-voltage measurement is taken. It is important for these high resistivity materials that the measurement is performed in high vacuum and in darkness. Both ambient moisture and stray light result in erroneous readings, by surface leakage and photoconductivity, respectively. The current-voltage measurement is performed sufficiently slowly that transient effects are not significant. Typically, the voltage is ramped in steps of $25 \mathrm{mV}$ at $1 \mathrm{~Hz}$ rate. Ramping from $-10 \mathrm{~V}$ to +10 is first done, followed by ramping from +10 to $-10 \mathrm{~V}$, to check for detector polarization and reproducibility.

After finishing the current-voltage measurement, the sample is biased at $-10 \mathrm{~V}$, and the cryostat is cooled, while the current is monitored. This is called the dark conductivity measurement, and it gives the conductivity and the resistivity of the sample as a function of the temperature. After a few hours of cooling, the sample temperature drops from room temperature to $\sim 20 \mathrm{~K}$.

At this point, the external tungsten-halogen lamp is turned on, and the photoconductivity of the sample is monitored. The excitation wavelength is scanned until the wavelength giving the maximum photoconductivity current is found. Then the sample is photoexcited at that wavelength for approximately 1 hour. By selecting the photoexcitation wavelength this way, the photoexcitation occurs in a relatively large volume of the detector. This is important, because, if the excitation wavelength is too short, then the photoexcitation will occur primarily at the surface, filling mostly the surface traps. If the wavelength is too long, then the photoexcitation will not photogenerate significant concentration of electrons and holes, resulting in negligible filling of the traps. For the CdZnTe samples we have studied, the excitation wavelengths for maximum photoconductivity currents are found to be between 600 to $800 \mathrm{~nm}$.

After photoexcitation for some duration, approximately 1 hour, the voltage bias across the sample is turned off, the external tungsten-halogen light is turned off, and the sample is kept in complete darkness. Then the sample is gradually heated in the dark. Here is essentially where the difference between thermally stimulated current (TSC) and thermoelectric emission spectroscopy (TEES) comes in. In TSC, the sample is gradually heated and the voltage bias is left on. Any carriers emitted from the traps in the sample are driven by the applied electric field and measured as the TSC current. Sometimes, He exchange gas is used to minimize the temperature gradient across the sample. However, in TEES, the voltage bias is off, and the heating rate is higher, resulting in a temperature gradient of a few degrees across the sample. In the absence of an applied electric field, this temperature gradient causes the carriers emitted from the traps to travel from the hot region to the cold region of the sample. Since electrons and holes have opposite charge polarities, their motion in the same direction give opposite signs of the currents. This means that, unlike TSC, TEES can be used to distinguish between electron and hole traps by noting the signs of the TEES currents. Also, since TEES is performed under zero voltage bias, it can be taken at higher temperatures, in order to study truly deep levels, where any result from TSC would be nearly indecipherable due to large leakage current from the applied voltage bias.

\section{EXPERIMENTAL RESULTS AND DISCUSSION}

In this paper, we discuss the results from a sample obtained from a commercial supplier of spectrometer-grade $\mathrm{CdZnTe}$ detectors. The sample is grown by the high-pressure Bridgeman method and it is typical of some of the samples we have studied. The sample dimensions are $10 \times 10 \times 2 \mathrm{~mm}^{3}$ and it has Au electrodes on its two $10 \times 10 \mathrm{~mm}^{2}$ surfaces. The current-voltage curve for this sample taken at $293 \mathrm{~K}$ is shown in Fig. 2. Note that the current-voltage curve is nearly linear. From the plot, the sample resistance is $1.6 \times 10^{10} \Omega$ and, taking into account the sample dimensions, the resistivity at $293 \mathrm{~K}$ is $7.9 \times 10^{10} \Omega \mathrm{cm}$.

The dark conductivity measurement is shown in Fig. 3, where the natural log of the current, under $10 \mathrm{~V}$ applied bias, is plotted vs. $\frac{1}{k T}$. The temperature range shown is from from $240 \mathrm{~K}$ to $293 \mathrm{~K}$. It can be seen that the current is related to $\frac{1}{k T}$ in an exponential manner with an activation energy of $695 \mathrm{meV}$. Note that the same activation energy is 
associated with both the conductivity and the resistivity of the sample, since these are related to the current by constant multiplication factors and power laws.

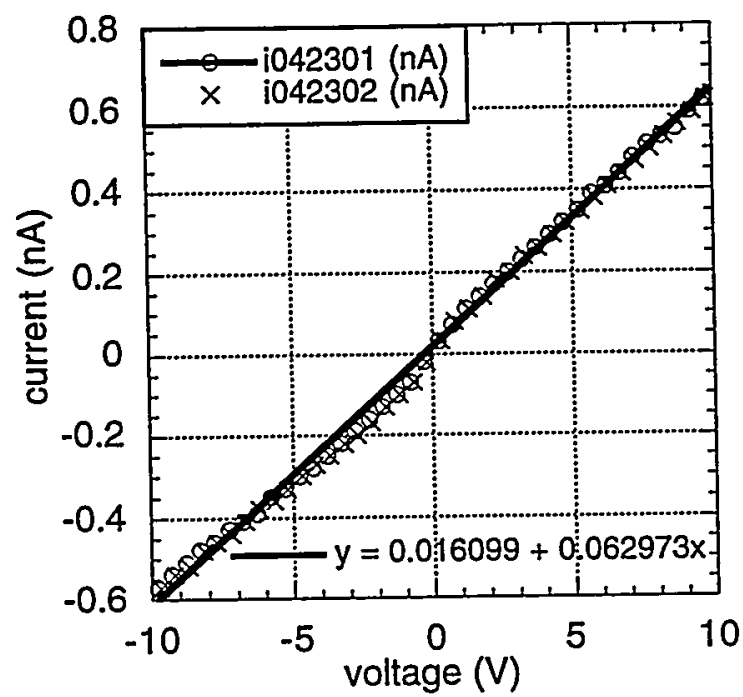

Fig. 2. Current-voltage measurements at $293 \mathrm{~K}$, in darkness and in high vacuum.

A linear fit is also shown along with its fit parameters.

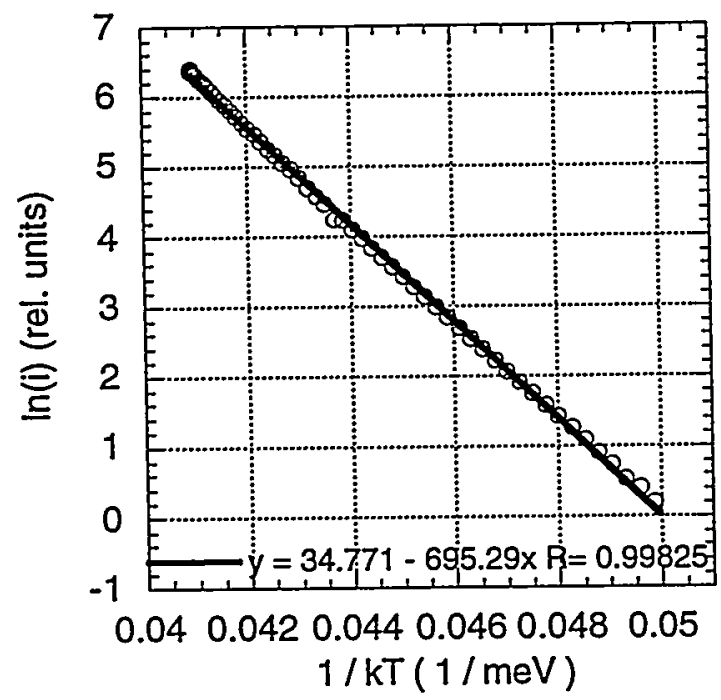

Fig. 3. Dark conductivity measurement, showing the natural log of the current at $10 \mathrm{~V}$ applied bias vs. $1 / \mathrm{kT}$. A linear fit is also shown along with its fit parameters.

For this sample, the photoexcitation at $\sim 20 \mathrm{~K}$ was done for $\sim 1$ hour at excitation wavelength of $720 \mathrm{~nm}$. The following shows the average temperature of the sample as a function of time during the subsequent heating of the sample. It can be seen that, after a few minutes, the heating rate becomes constant at $\sim 0.14 \mathrm{~K} / \mathrm{sec}$. 


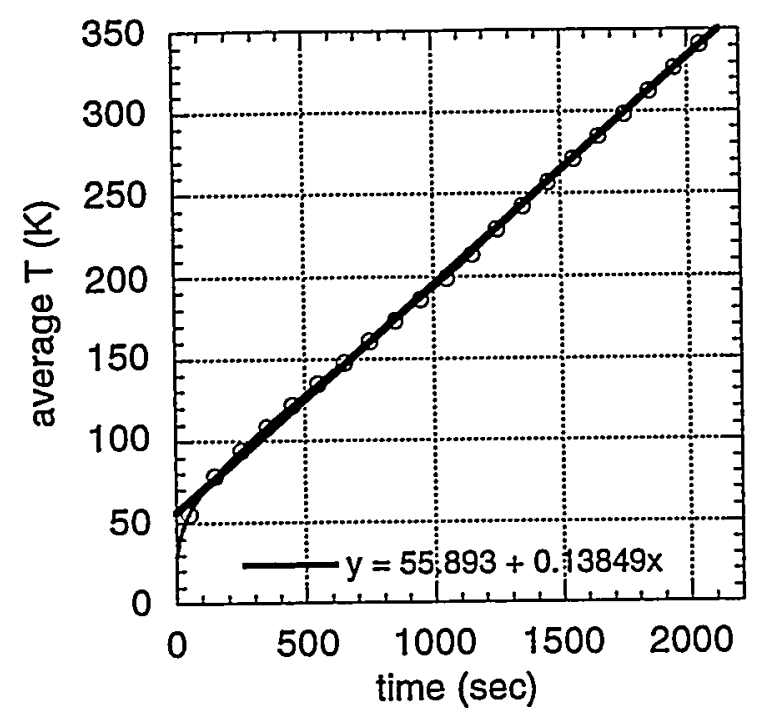

Fig. 4. The sample temperature vs. time. The time equals zero when heating is started at $20 \mathrm{~K}$.

The TEES run of this sample gave evidence of 1 electron trap and three hole traps. The TSC spectra, in general, show the same peaks, and they will not be shown here. The TEES spectrum is shown in Fig. 5. Clearly, an electron peak centered at $253 \mathrm{~K}$ can be seen, and three hole peaks at 33,50 , and $281 \mathrm{~K}$ can be seen as well.

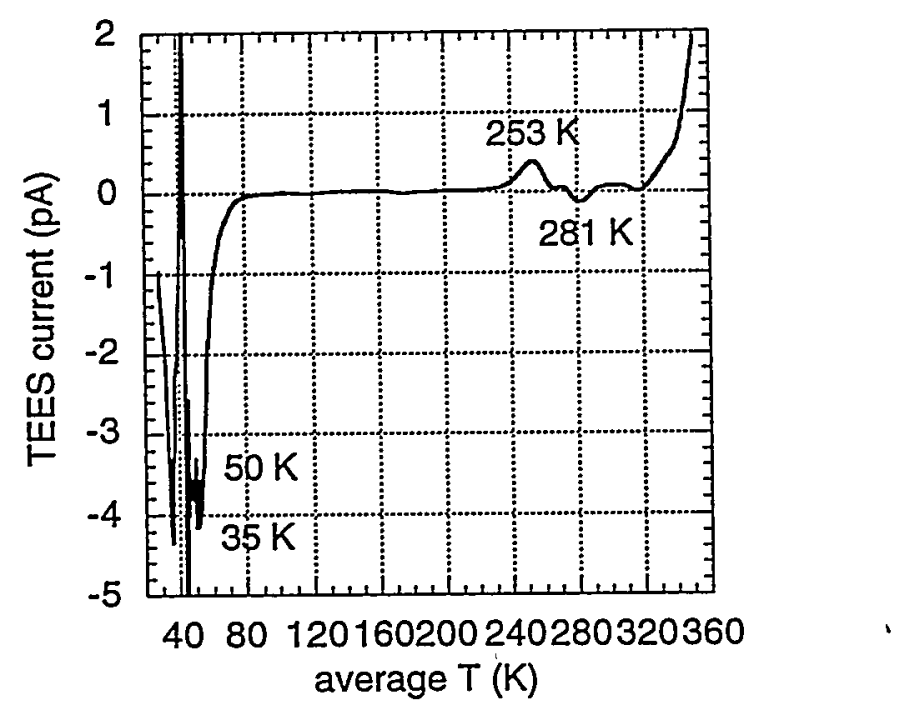

Fig. 5. The TEES spectrum. In the above, a positive TEES current is due to emission of electrons from electron traps in the sample and a negative TEES current is due to emission of holes from hole traps in the sample.

For the electron peak at $253 \mathrm{~K}$, it is possible to extract the activation energy for this trap directly from the above data. This is based on the fact that the initial rise of the TEES current is exponentially dependent upon the trap energy ${ }^{8}$. Fig. 6 shows a plot of the TEES current immediately below the $253 \mathrm{~K}$ peak vs. $\frac{1}{k T}$. 


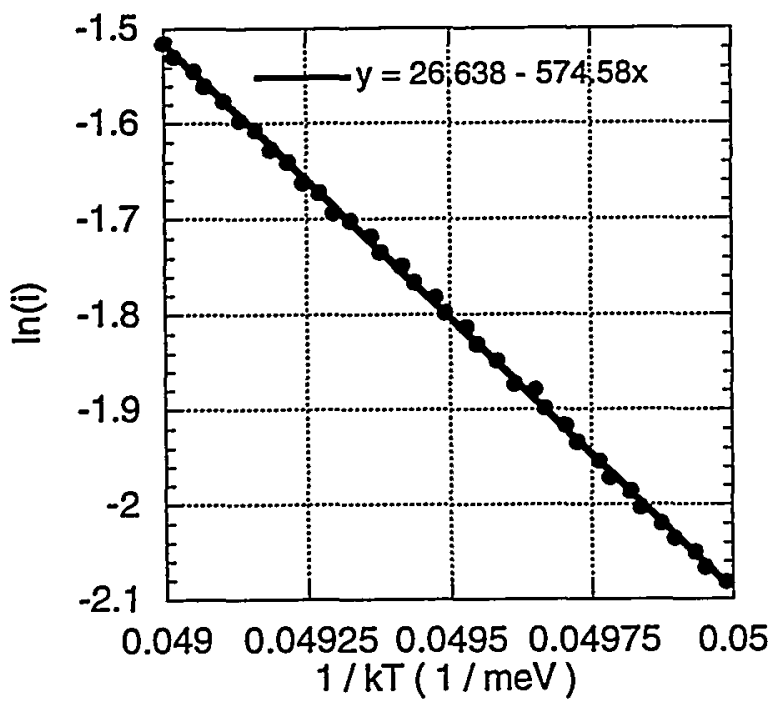

Fig. 6. The early rise portion of the electron trap peak centered at $253 \mathrm{~K}$.

A linear fit is also shown with its fit parameters.

The slope of the linear fit is the trap energy, and it is found to be $575 \mathrm{meV}$. Using this and the following formula ${ }^{9}$, the trap cross section can be calculated. The formula was originally developed for TSC, but it is now commonly used for both TSC and TEES.

$$
E_{t}=k T_{m} \ln \left(\frac{c m_{e}^{*} \sigma_{e} E_{t} T_{m}^{4}}{\beta}\right) \text {, where } c=\frac{4 \sqrt{6 \pi^{3}} k^{3}}{h^{3}} . \quad \text { Eq. (1) }
$$

In the above equation, $E_{t}$ is the trap energy, $k$ is the Boltzmann constant, $T_{m}$ is the temperature at the peak, $m_{e}^{*}$ is the effective mass of electrons, $\sigma_{e}$ is the capture cross section, $\beta$ is the heating rate, and $h$ is the Planck's constant. For $m_{e}^{*}$, we use $0.14 m_{e}$, where $m_{e}$ is the free electron mass. This is the known effective mass for CdTe and we can use this, because the $\mathrm{Zn}$ concentrations in the samples we encounter are relatively low, being in the rage from 0.1 to 0.3 . Using Eq. (1), we obtain $8 \times 10^{-16} \mathrm{~cm}^{2}$ as the capture cross section for the electron trap.

The other peaks in the TEES spectrum overlap, and hence the initial rise method can not be used directly. However, one can use Eq. (1) to estimate the trap energy. Realistically, the capture cross section is expected to be between $10^{-12}$ to $10^{-18} \mathrm{~cm}^{2}$. A negatively charge electron trap or a positively charge hole trap would have a small cross section, and a positively charged electron trap or a negatively charge hole trap would have a large cross section. Propagating the range of uncertainties in $\sigma$ to uncertainties in $E_{t}$, Eq. (1) gives $70 \pm 20 \mathrm{meV}, 105 \pm 30 \mathrm{meV}$, and 694 $\pm 162 \mathrm{meV}$. Note that the 6 orders of magnitude in the uncertainty of $\sigma$ corresponds to only $\sim 30 \%$ uncertainty in the trap energies, due to the logarithmic dependence of the trap energy on the trap cross section.

\section{MODELING}

In the previous section, we have seen that there is one electron trap and three hole traps in the sample. By detailed analysis, we obtained the trap energies and, for the electron trap, the trap cross section was obtained as well. Now let us construct a model of how a CdZnTe crystal containing these traps should behave. The model is based on the following energy level diagram. The energies of the trap levels and their types (electron or hole) are determined by the TEES result shown previously. The electron trap is designated E1, and the hole traps, going from the deepest to the shallowest, are designated $\mathrm{H1}, \mathrm{H} 2$, and $\mathrm{H} 3$. The concentration of the traps are used as the fitting parameters in this model. The value of $1.572 \mathrm{eV}$ is the known energy band gap of $\mathrm{Cd}_{0.9} \mathrm{Zn}_{0.1} \mathrm{Te}$ at $300 \mathrm{~K}$. We assume that the sample has $0.1 \mathrm{Zn}$ concentration, based on previous studies of similar material. 


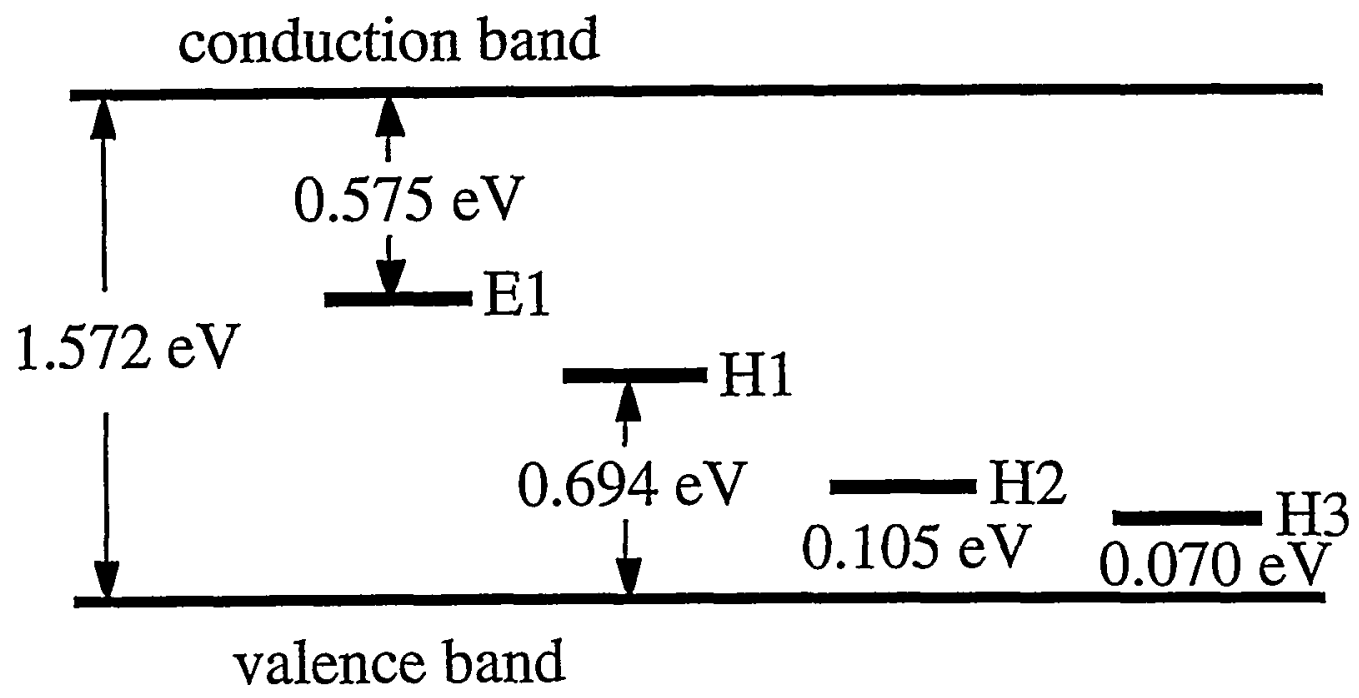

Fig. 7. The energy level diagram of the sample, showing an electron trap E1 and three hole traps, $\mathrm{H} 1, \mathrm{H} 2$, and $\mathrm{H} 3$, all within the energy band gap of $\mathrm{CdZnTe}$.

For simplicity, we assume that the cross sections for all the traps are the same and have the value of $8 \times 10^{-36}$ $\mathrm{cm}^{2}$, the value for the electron trap E1. Let the trap concentration of E1 be $10^{15} \mathrm{~cm}^{-3}$ and the trap concentration of $\mathrm{H} 1$ be $0.33 \times 10^{15} \mathrm{~cm}^{-3}$. The relative trap concentrations are determined by the 3 to 1 ratio of the TEES peaks for E1 and $\mathrm{H} 1$ shown in Fig. 5. The absolute magnitudes of the trap concentrations are educated guesses. For $\mathrm{H} 2$ and $\mathrm{H} 3$, we assume trap concentrations of $0.34 \times 10^{15} \mathrm{~cm}^{-3}$ each. It will be seen that, using these trap concentrations, our model quantitatively describes the observed electrical resistivity at room temperature and the temperature dependence of the resistivity. Additionally, we assume that the traps are neutral when filled.

In our model, given the energy band diagram shown in Fig.7, the electrical property of the material is determined by the free electron and the hole concentrations consistent with semiconductor physics. At any given temperature, the traps are partially filled, due to ionization by thermal energy. Ionized traps are charged and attract oppositely charged carriers. We require that the charge neutrality is maintained, i.e. the sum of the number of the positively charged traps and the number of holes equals the sum of the number of the negatively charge traps and the number of electrons. For the case sjpwm in Fig. 7, the following equation must hold to preserve the charge neutrality.

$$
N_{E 1}^{+}+p=N_{H 1}^{-}+N_{H 2}^{-}+N_{H 3}^{-}+n, \quad \text { Eq. (2) }
$$

where the charged trap concentrations are determined by the Fermi-Dirac statistics and $n$ and $p$ are the free electron and hole concentrations. In addition, the law of mass action must hold.

$$
p n=n_{i}^{2}, \quad \text { Eq. (3) }
$$

where $n_{i}$ is the intrinsic carrier concentration. Recall that $n$ and $p$ are related to the Fermi level by the following formula, for nondegenerate semiconductor, like our sample.

$$
n=N_{c} \exp \left(-\frac{E_{c}-E_{f}}{k T}\right) \text { and } p=N_{\nu} \exp \left(-\frac{E_{f}-E_{c}}{k T}\right), \text { Eq. (4) }
$$

where $N_{c}$ and $N_{v}$ are the effective density of the states for the conduction band and the valence band. For CdTe, these are $1.3 \times 10^{18} \mathrm{~cm}^{-3}$ and $5.2 \times 10^{18} \mathrm{~cm}^{-3}$ at $300 \mathrm{~K}$, respectively. Note that these have implicit $\mathrm{T}^{3 / 2}$ dependence. Using Eq. (3), $p$ can be eliminated from Eq. (2). Furthermore, using Eq. (4), $n$ can be expressed as a function of $E_{f}$. Then the Eq. (2) becomes a nonlinear equation in $E_{f}$, only, and $E_{f}$ can be determined by using a numerical root finding algorithm. Once $E_{f}$ is known, the carrier concentrations can be calculated using Eq. (4).

Performing the computation outlined above, we find that $E_{f}$ is $0.797 \mathrm{eV}$ above the valence band maximum at $293 \mathrm{~K}$ for our sample. This gives free electron and hole concentrations of $5.9 \times 10^{4} \mathrm{~cm}^{-3}$ and $1.58 \times 10^{5} \mathrm{~cm}^{-3}$. Note that 
the hole concentration is actually larger than the electron concentration. However, because the electron mobility is $\sim 10$ times larger than the hole mobility, the conductivity, $\sigma=e \mu_{e} n+e \mu_{h} p$, is dominated by the electron contribution. For example, the typical electron mobility in CdZnTe is $1000 \mathrm{~cm}^{2} / \mathrm{V} / \mathrm{sec}$ and the typical hole mobility is $100 \mathrm{~cm} / \mathrm{V} / \mathrm{sec}$. Using these values, the conductivity is calculated. The resistivity is just its reciprocal, and it is calculated to be $8.0 \times 10^{10}$ $\Omega \mathrm{cm}$, which is in agreement with the measured value of $7.9 \times 10^{10} \Omega \mathrm{cm}$ stated earlier.

The model can be used also to predict the resistivity of the sample as a function of its temperature. The result is shown in Fig. 8. The activation energy can be seen to be $780 \mathrm{meV}$. This is larger than the measured value of $695 \mathrm{meV}$ only by approximately $12 \%$.

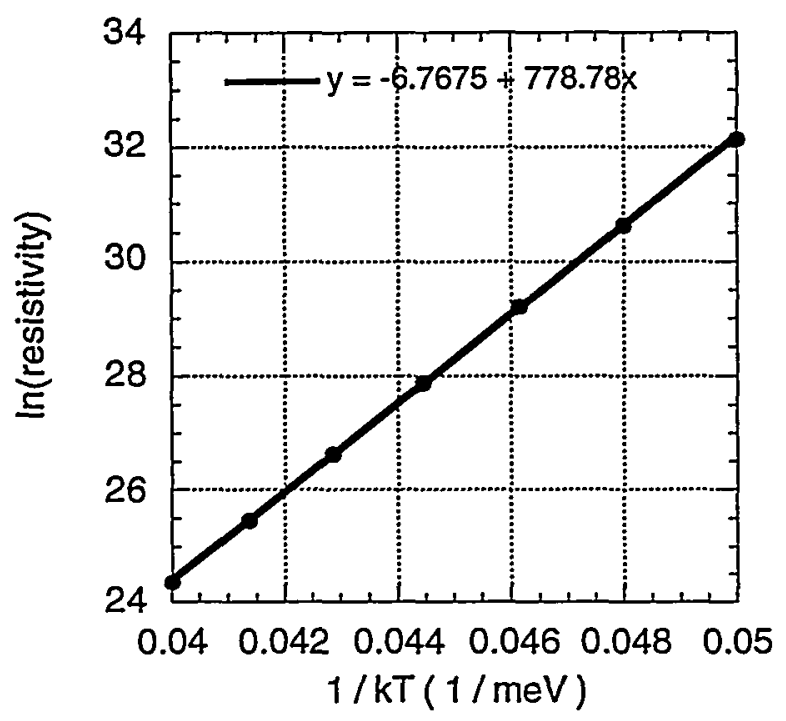

Fig. 8. Model prediction of resistivity as a function of temperature. The circles are predicted resistivities and the line is a linear fit. The fit parameters are also shown.

\section{CONCLUSION}

In summary, we have detected traps in CdZnTe radiation detectors by thermoelectric emission spectroscopy and thermally stimulated conductivity. Detailed analysis of the data allows us to find the trap energies, and, in some cases, the trap cross sections. We have developed models of trapping and charge compensation in these materials, to predict the electrical resistivity based on the trap energies. By using reasonable trap concentrations as fit parameters, we are able to reach excellent agreements with the actual electrical properties

In the future, we will search for more traps and further develop our models. In addition, we hope to find the relation of these to the actual radiation detector performance and to the compositional and structural properties of the materials. Ultimately, we want to control the traps to engineer the materials for optimum performance as radiation detector materials.

\section{ACKNOWLEDGMENTS}

This work has been funded by the U. S. Department of Energy contract no. DE-AC04-94AL85000 and also by the U. S. Department of Energy, Office of Nonproliferation and National Security. We would like to thank Professor Kelvin Lynn of Washington State University for useful conversations during the early portion of this work. 


\section{REFERENCES}

1. For a recent review, see Semiconductors for Room-Temperature Radiation Detector Applications, Proceedings of Materials Research Society Symposium, Vol. 302, edited by R. B. James, T. E. Schlesinger, Paul Siffert, and Larry Franks (Materials Research Society, Pittsburgh, Pennsylvania, 1993).

2. B. Santic and U. V. Desnica, Appl. Phys. Lett. 56, 2636(1990).

3. Z. C. Huang, K. Xie, and C. R. Wie, Rev. Sci. Instrum. 62, 1951(1991).

4. Z. C. Huang, E. Eissler, and C. R. Wie, Nucl. Instr. and Meth. B 100, 507(1995).

5. Cs. Szeles, Y. Y. Shan, K. G. Lynn, A. R. Moodenbaugh, and E. E. Eissler, Phys. Rev. B 55, 6945(1997).

6. Cs. Szeles, Y. Y. Shan, K. G. Lynn, and E. E. Eissler, Nucl. Instr. and Meth. A 380, 148(1996).

7. D. Bloom and S. W. S. McKeever, J. Appl. Phys. 77, 6521(1995).

8. Gustavo A. Dussel and Richard H. Bube, Phys. Rev. 155, 764(1967); It is implicitly assumed that the TEES current is linearly proportional to the free carrier concentration. We have recently shown this. E. Y. Lee and R. B. James (to be published).

9. R. H. Bube, Photoconductivity in Solids (Wiley, New York, 1960). 